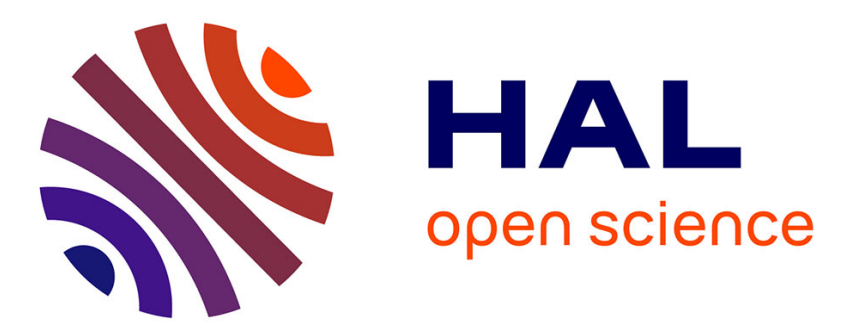

\title{
Two varieties of conditionals and two kinds of defeaters help reveal two fundamental types of reasoning
}

\author{
Guy Politzer, Jean-François Bonnefon
}

\section{To cite this version:}

Guy Politzer, Jean-François Bonnefon. Two varieties of conditionals and two kinds of defeaters help reveal two fundamental types of reasoning. Mind and Language, 2006, 21 (4), pp.484-503. ijn_00142406

\section{HAL Id: ijn_00142406 \\ https://hal.science/ijn_00142406}

Submitted on 18 Apr 2007

HAL is a multi-disciplinary open access archive for the deposit and dissemination of scientific research documents, whether they are published or not. The documents may come from teaching and research institutions in France or abroad, or from public or private research centers.
L'archive ouverte pluridisciplinaire HAL, est destinée au dépôt et à la diffusion de documents scientifiques de niveau recherche, publiés ou non, émanant des établissements d'enseignement et de recherche français ou étrangers, des laboratoires publics ou privés. 
Mind and Language, 2006, 21(4), 484-503.

\title{
Two varieties of conditionals and two kinds of defeaters help reveal two fundamental types of reasoning
}

\author{
Guy Politzer \\ Institut Jean-Nicod, CNRS \\ Jean-François Bonnefon \\ University of Toulouse-2
}

Two notions from philosophical logic and linguistics are brought together and applied to the psychological study of defeasible conditional reasoning. The distinction between disabling conditions and alternative causes is shown to be a special case of Pollock's (1987) distinction between 'rebutting' and 'undercutting' defeaters. 'Inferential' conditionals are shown to come in two varieties, one that is sensitive to rebutters, the other to undercutters. It is thus predicted and demonstrated in two experiments that the type of inferential conditional used as the major premise of conditional arguments can reverse the heretofore classic, distinctive effects of defeaters.

The authors are grateful to Didier Dubois for helpful technical suggestions. 


\section{Introduction}

Fifteen years of psychological experiments have firmly established the fact that sound inferences can be defeated by the provision of additional information: A conclusion $C$ that has been derived from a set of beliefs $B$ can be retracted upon the arrival of some additional premise $A .{ }^{1}$ Psychological research has focused in particular on four basic arguments, which usually are referred to as conditional reasoning arguments. While researchers may have different theoretical takes on how this reasoning operates, a wide consensus obtains about a Core Pattern of results, to which we will return shortly; in a nutshell, the two conditional arguments that are deductively valid are defeated by 'disabling conditions', whereas the two invalid ones are defeated by 'alternative causes' (or 'alternative antecedents').

\footnotetext{
${ }^{1}$ It is unclear whether these experiments demonstrate the nonmonotonic nature of human reasoning, in the technical sense, and we will thus avoid using that term (we are indebted to an anonymous referee for warning us against that possible confusion). Strictly speaking, reasoning is nonmonotonic when a valid inference can be turned invalid with the provision of new information. Psychological experiments rather defeat a conclusion by manipulating the credibility of the premises that if true would lead to that conclusion. It will be clear later in this article that such is the approach we adopt to our own results.
} 
In this article we will prove this consensus somewhat hasty, as we will show how to obtain, in some specific circumstances, a complete reversal of this pattern of results, elaborating theoretically and experimentally on a and by Thompson (1914) result obtained by Cummins (1995)/ Drawing on linguistics and philosophical logic, we will propose a clear pattern of relationships between two types of 'inferential' conditionals (which will be defined), two kinds of defeaters (rebutting and undercutting), and two categories of conditional arguments (deductively valid and deductively invalid). We begin however with a presentation of the Core Pattern of results, listing some of the many publications that support it.

\section{Psychological Background: The Core Pattern of Results}

Two distinct yet interwoven experimental paradigms have addressed the question of how conditional inferences can be 'suppressed', or, as we will also write, how the conclusions of conditional arguments can be defeated. Both paradigms focus on the endorsement rate (or mean perceived likelihood) of the conclusions of the four basic conditional arguments: The two deductively valid arguments, Modus Ponens (MP: If A then B; A; therefore, B) and Modus Tollens (MT: If A then B; not B; therefore, not A), and the two deductively invalid arguments, Affirmation of the Consequent 
(AC: If A then B; B; therefore, $A$ ) and Denial of the Antecedent (DA: If A then $B$; not $A$; therefore, not B).

Both paradigms have also made use of the notions of disabling conditions and alternative causes borrowed from the literature on causality. Consider the causal relation between eating a lot of candy (the cause) and having cavities (the effect). A factor that makes it possible for the effect to be absent whilst the cause is present is a disabling condition to this relation: Superb dental hygiene is a disabling condition to the relation between candy and cavities. Comparably, a factor that makes it possible for the effect to be present whilst the cause is absent is an alternative cause (or alternative condition): Abysmal dental hygiene is an alternative cause of cavities, independent of compulsive candy eating.

The first paradigm (henceforth the overt paradigm) has focused on the effect of introducing overtly a cue to the existence of disabling conditions or alternative causes on the endorsement rate of MP, MT, DA and AC from a major conditional premise. The cue has traditionally taken the form of an additional conditional statement such as (2) below which, added to an MP argument whose premises are (1) and (3), yields Byrne's (1989) well-known library example:

(1) If she has an essay to write then she will study late in the library;

(2) If the library stays open then she will study late in the library; 
(AC: If A then B; B; therefore, $\mathrm{A}$ ) and Denial of the Antecedent (DA: If A then $B$; not $A$; therefore, not $B$ ).

Both paradigms have also made use of the notions of disabling conditions and alternative causes borrowed from the literature on causality. Consider the causal relation between eating a lot of candy (the cause) and having cavities (the effect). A factor that makes it possible for the effect to be absent whilst the cause is present is a disabling condition to this relation: Superb dental hygiene is a disabling condition to the relation between candy and cavities. Comparably, a factor that makes it possible for the effect to be present whilst the cause is absent is an alternative cause (or alternative condition): Abysmal dental hygiene is an alternative cause of cavities, independent of compulsive candy eating.

The first paradigm (henceforth the overt paradigm) has focused on the effect of introducing overtly a cue to the existence of disabling conditions or alternative causes on the endorsement rate of MP, MT, DA and AC from a major conditional premise. The cue has traditionally taken the form of an additional conditional statement such as (2) below which, added to an MP argument whose premises are (1) and (3), yields Byrne's (1989) well-known library example:

(1) If she has an essay to write then she will study late in the library;

(2) If the library stays open then she will study late in the library; 
(3) She has an essay to write.

Premise (2) is a cue to the existence of a disabling condition (i.e., the library not staying open late) to the relation between having an essay to write and working late at the library. When premise (2) is introduced alongside premises (1) and (3), the endorsement rate of the MP conclusion 'she will study late in the library' drops dramatically.

From Rumain, Connell, and Braine (1983) to Politzer (2005), through numerous replications and extensions (Bonnefon \& Hilton, 2002; Chan \& Chua, 1994; Dieussaert, Schaeken, Schroyens, \& d'Ydewalle, 2000; Manktelow \& Fairley, 2000; Politzer \& Bourmeau, 2002; Stevenson \& Over, 1995 - see also Bonnefon \& Hilton, 2004; Oaksford \& Chater, 2003), it has been established that cueing to the existence of a disabling condition decreases confidence in the conclusion of the valid MP and MT arguments ('she will study late in the library' in the example above). And similarly, cueing to the existence of an alternative condition decreases confidence in the invalid conclusions of the AC and DA arguments. That is, borrowing another standard example from Byrne (1989), for an AC argument whose premises are (4) and (6), adding premise (5) below decreases the rate of endorsement of the conclusion 'she has an essay to write') :

(4) If she has an essay to write then she will study late in the library; 
(5) If she has some textbooks to read then she will study late in the library;

(6) She will study late in the library.

Those results have found echo in a large number of studies conducted within a second paradigm, which we call the covert paradigm, which aims at demonstrating that disablers and alternatives have an effect on conditional inferences even when the reasoner is not overtly reminded about them. From Cummins, Lubart, Alksnis, and Rist (1991) to De Neys, Schaeken, and d'Ydewalle (2003), numerous studies (e.g., Cummins, 1995; De Neys, Schaeken, and d'Ydewalle, 2002; Markovits, 1986; Markovits \& Quinn, 2002; Quinn \& Markovits, 1998; Thompson, 1994,1995) have demonstrated that (a) the higher the tendency for a conditional to evoke alternative conditions, the lower the endorsement rate of DA and $\mathrm{AC}$, and (b) the higher the tendency for a conditional to evoke disabling conditions, the lower the endorsement rate of MP and MT. For example, DA and AC arguments have lower endorsement rates of their conclusion with a major premise such as 'if Mary jumped into the swimming pool then she got wet' (many alternative causes) than with 'if Joe cut his finger then it bled' (few alternative causes). Similarly, MP and MT arguments have lower endorsement rates of their conclusion with the rule 'if the match was struck then it lit' (many disabling 
conditions) than with 'if the gong was struck then it sounded' (few disabling conditions).

Thus, the two experimental paradigms concur to what we call here the Core Pattern of results: Disabling conditions defeat the conclusions of MP and MT (but usually not the conclusions of DA and AC) and alternative conditions defeat the conclusion of DA and AC (but usually not the conclusions of the valid MP and MT). This Core Pattern is endorsed by most if not all researchers in the field, and, apart from the occasional breach (see in particular Markovits \& Potvin, 2001), has never been seriously questioned. Notice that in all this literature, disabling conditions and alternative causes are attached to the antecedent part of the conditional sentence.

Nevertheless, an important twist to the Core Pattern is due to Thompson(1994) and Cummins (1995), though it has not yet received the attention it deserves. Cismmins claimed that, in the causal domain, alternative causes cast doubt on the necessity of the cause for bringing about the effect, and that disabling conditions cast doubt on the sufficiency of the cause. Clearly, this analysis depends only on the identification of the cause and of the effect, and is therefore independent of the syntactic form of the causal rule. Cummins thus predicted that should a rule following the standard 'if cause, then effect' form (e.g., 'if the brake was depressed then the car slowed down') be 
changed into the reverse form 'if effect, then cause' (e.g., 'if the car slowed down then the brake was depressed'), disabling conditions and alternative causes would keep similar effects as they have with the direct conditional form - thus suggesting a possible reversion of the Core Pattern. This is because turning an initial argument such as, e.g., MP 'if [cause] then [effect]; [cause]; therefore [effect]' into 'if [effect] then [cause]; [effect]; therefore [cause]', amounts to turning the initial MP argument into an $\mathrm{AC}$ argument although formally it remains an MP argument (and similarly for MT in regard to DA).

This prediction was clearly supported by the results of an experiment that used the covert paradigm. We believe that these results call for further investigation, both experimental and conceptual.

Experimentally, one has to make sure that Cummins's results are not limited to the covert paradigm. One question that the present study will address is whether the results also obtain for the overt paradigm; only in case of a positive answer could the result be considered robust, albeit still limited to causality. The other question that will be addressed is whether the same results obtain for relationships other than causal.

Theoretically, it will be shown that Cummins's (1995; Cummins, Lubart, Alksnis, \& Rist, 1991) conceptualization in terms of disablers and alternatives on the one hand, and the use of reversed conditionals on the 
other hand can be linked with work in philosophical logic and in linguistics, respectively, which will shed new light on the use and nature of conditional arguments. We begin with Pollock's speculations on the two ways to defeat a valid argument.

\section{Philosophical Background: Two Routes to Defeasible Reasoning}

Pollock (1987) used the term defeater to designate any reason that may lead to the revocation of some conclusion. Given a reason $\mathrm{A}$ to believe $\mathrm{C}$, a defeater of the inference from $A$ to $C$ is a reason $D$ which is (a) consistent with $A$, but such that (b) A and D is not a reason to believe C. (In order to differentiate between defeasible and nondefeasible cases, Pollock defined defeaters in terms of prima facie reasons, but we need not use this distinction for our current purpose.)

For example, consider the situation where Alice is in her car and turns the switch (A). This is a reason to believe that the engine will start (C). Now the battery being disconnected (D) is not inconsistent with Alice turning the switch, yet the conjunction of Alice turning the switch and the battery being disconnected (A and $\mathrm{D}$ ) is not a reason to believe that the engine will start. It would rather count against the conclusion that the engine will start. This is what Pollock called a rebutting defeater: $\mathrm{D}$ is rebutting defeater to the 
inference from $A$ to $C$ when $A$ and $D$ together no longer form a reason to believe $C$, or even form a reason to believe not- $C$ rather than $C$.

The disabling conditions that have been shown to defeat MP and MT are by definition rebutting defeaters: a disabling condition $\mathrm{D}$ is a factor that may prevent the effect $(C)$ from occurring whilst the cause (A) is present - in other words, $A$ is a reason to believe $C$, but $A$ and D together do not form a reason to believe $C$, or even form a reason to believe not $C$. Thus, the Core Pattern concurs so far to Pollock's speculations. However, Pollock considered a second kind of defeater, which does not fit so straightforwardly in the Core Pattern.

Alongside rebutting defeaters, Pollock (1987) defined undercutting defeaters. Undercutting defeaters do not attack the conclusion $C$ itself, but rather the connection between $\mathrm{A}$ and $\mathrm{C}$. An undercutting defeater $\mathrm{D}$ is a reason for denying that $A$ would not be true unless $C$ were true. Pollock $s$ example is the following: Some object looks red to you (A), which is a reason to believe that it is red (C). But you discover that it is illuminated by red light (D). D is a defeater, because you no longer have solid ground to believe that the object is red. It is not a rebutting defeater, for the object being illuminated by red light is not a reason to believe that it is not red - rather, it is the claim that the object would not look red unless it were red that is 
refuted by the fact that the object is illuminated by red light. $D$ is thus an undercutting defeater.

Undercutting defeaters bear some striking resemblance to alternative causes: Being illuminated by red light is indeed an alternative cause for looking red, besides being red. Thus, Pollock's example of undercutting defeat illustrates how an alternative cause can defeat, not an invalid argument as exhibited in the Core Pattern, but a valid one, namely Modus Ponens. Indeed, MP applied to premises (7) and (8) below would lead to the conclusion 'the object is red', yet this conclusion would be defeated by introducing the alternative cause featured in the antecedent of premise (9):

(7) If an object looks red, then it is red;

(8) This object looks red;

(9) If an object is illuminated by red light, then it looks red.

Now, the key element of Pollock's example appears to be the peculiar nature of the conditional statement in (7). Although this statement contains the cause and the effect of a causal relation, it is not framed in a standard 'if cause then effect' form, nor does it express such a relation. Rather, it is framed in the reversed conditional form, Philosophical logic thus provides independent theoretical justification for the need to consider how reversed conditionals can reverse the Core Pattern 
of psychological results. However, there is more to say about these reversed conditionals when one adopts a linguistic point of view.

\section{Linguistic Background: Inferential Conditionals}

Causal conditionals have a cause as their antecedent and its effect as their consequent (e.g., 'If an object is red, then it looks red'), and not the opposite. ${ }^{2} \quad$ Linguists would actually qualify the conditional in (7) as an example of epistemic conditional (Dancygier, 1998; Sweetser, 1990) or, in another terminology, of inferential conditional (Dancygier, 1993)

Inferential conditionals express that knowledge of the truth of the propositional content of the antecedent allows the speaker to infer the truth of the propositional content of the consequent (with an appropriate degree of confidence). Elaborating on Werth (1997), we distinguish two types of inferentials, which we will now consider in turn: (a) necessary epistemic conditionals, which appear to work as a compacted Modus Ponens inference, and belong to the realm of deductive inference; and (b) possible epistemic conditionals, a transformation of the former, which belong to the realm of non demonstrative inference. Here are three examples of necessary epistemic conditionals:

2. A form which Pearl (1988) calls an evidential conditional. 
(10) If Harry has an alibi, (then) he is not the murderer;

(11) If the brake was depressed (then) the car slowed down;

(12) If Julie has a presentation to prepare (then) she is in the library.

In all these examples, knowledge of the truth of the antecedent is assumed by the speaker, and confidence in the conclusion reaches certainty (providing there is no rebutter, but this is not yet our concern). In (10) the practical notion that someone with an alibi cannot be the murderer is directly applied to clear Harry with certainty. In (11) knowledge of a causal law is applied to explain an event which follows with practical certainty. Finally in (12) knowledge of Julie's means to reach her aim warrants her to be in the library.

In brief, these inferential conditionals are some kind of compacted Modus Ponens used to deliver certain conclusions. They can be paraphrased in at least two characteristic ways: (a) by adding 'obviously' or 'necessarily' in the consequent after 'then', or (b) by adding 'cannot' to negative consequents, (e.g., 'if Harry has an alibi, then he cannot be the murderer') and 'must' to positive consequents (e.g., 'if the brake was depressed then the car must have slowed down'). In that case, 'must' has a necessity reading. Our second type of inferential conditionals (where confidence in the inference does not reach certainty) is obtained by switching antecedent and 
consequent of conditionals of the first type, as shown by the following examples:

$\left(10^{\prime}\right)$ If Harry is not the murderer (then) he has an alibi;

(11') If the car slowed down (then) the brake was depressed;

$\left(12^{\prime}\right)$ If Julie is in the library (then) she has a presentation to prepare.

It is essential to note the uncertain status of the truth of the consequent, given knowledge of the truth of the antecedent. In $\left(10^{\prime}\right)$, upon knowing that Harry is not the murderer, one has good reason to believe that he provided an alibi, although one may have no alibi while being innocent, hopefully. In (11'), putting on the brake is the most likely reason for slowing down, although not the only possible one. In (12'), knowledge of Julie's habits may suggest that preparing a presentation is the best justification for her being in the library but this does not exclude other possible reasons. As earlier, a paraphrase of these sentences can be obtained in several ways. One is to insert 'it means that' in the consequent after (or better, instead of) 'then'. This paraphrasing is the main characterization of this type of inferential conditional. Another way is to nuance confidence in the consequent by an appropriate modal: possibly, probably, etc. Yet another way is to add, as earlier, the word 'must' in the consequent - yet it is essential to note that in the present case, 'must' does not take a necessity reading, but a possibility reading; it serves the same purpose as the second 
paraphrasing device, namely to communicate that the consequent cannot be inferred to be certainly true. In brief, with this second variety of inferential conditional, knowledge of the truth of the antecedent warrants some degree of belief in the consequent.

We have distinguished two types of inferential conditionals which are dual to each other: Necessary epistemic conditionals lead the speaker to infer the consequent as certain, usually presupposing the truth of their antecedent. They belong to demonstrative inference. Possible epistemic conditionals lead the speaker to infer the consequent with some level of confidence. They belong to the domain of non demonstrative inference. We have noticed that necessary epistemic conditionals work as some sort of compacted Modus Ponens; we defer to the general discussion a similar characterization of possible epistemic conditionals. We will now reconsider Pollock's example of undercutting defeat and Cummins's (1995) results in light of the distinction we have just introduced, and derive from there the experimental predictions that we will test in this article.

\section{Theoretical Integration and Experimental Predictions}

We can now requalify Pollock's example (7) as a possible epistemic inferential conditional featuring a causal relationship. Thus, Cummins's 
experimental manipulation (that switched the cause-effect conditional into its reversed formulation), and Pollock's definition of undercutting defeaters for MP (by way of using a conditional that reverses the cause-effect direction) concur to the same result: Valid arguments such as MP can be defeated by undercutting defeaters (alternative causes) when they operate on possible-epistemic inferential conditionals featuring causal relationships.

Interestingly, while Cummins's interest is explicitly in causal reasoning, Pollock did not set for himself such limits; yet his example belongs to this domain, presumably because causal reasoning together with the conditional rules that are used to express it, are prototypical of defeasible reasoning. Obviously, it is not exclusive, which is why in what follows we will use Pollock's more neutral terminology (rebutters and undercutters) rather than Cummins's more causality laden terms (disablers and alternative causes).

Indeed, it should be clear from the examples given in the previous section that possible-epistemic inferentials are not limited to causal relations. The linguistic framework we have introduced suggests that the transformation from necessary- to possible-epistemic inferential (by switching antecedent and consequent) can apply to a variety of relationships. In what follows, we will only consider one of them, namely 
the means-end relationship, as it has often been chosen in the psychological literature. We are now in a position to spell out our hypotheses:

Using the overt paradigm, when a possible-epistemic causal conditional is used as major premise of a conditional reasoning argument, we predict that introducing an undercutter will decrease the confidence in the conclusion of MP (P1) and of MT (P2), not affect the confidence in the conclusion of $\mathrm{AC}(\mathrm{P} 3)$ and of DA (P4). We predict that introducing a rebutter will not affect the confidence in the conclusion of MP (P5), and of MT (P6), and decrease the confidence in the conclusion of $\mathrm{AC}$ (P7) and of DA (P8). This set of eight predictions generalizes Cummins's results to the overt paradigm.

Besides, still using the overt paradigm, we predict that the same pattern will obtain when a possible-epistemic means-end conditional is used as the major premise of a conditional reasoning argument (hypotheses P1' to $\left.\mathrm{P}^{\prime}\right)$. This additional set of eight predictions is a further generalization from causal to non-causal possible-epistemic inferential conditionals.

All these predictions, expressed on the condition that the major conditional premise should be a possible-epistemic conditional, are the exact opposite to the Core Pattern. With classic causal conditionals, the conclusions of MP and MT are defeated by rebutters (in causal terms, 
disabling conditions), and the conclusions of DA and AC are defeated by undercutters (in causal terms, alternative conditions).

\section{Experiment 1}

\subsection{Method}

6.1.1. Participants A total of 131 participants were approached in Experiment 1 (mean age $=28.5, S D=11.4)$. Eighty-two of them had received college education or an equivalent, 21 had graduated from high school without pursuing further education, 26 had not graduated from high school, and 2 failed to provide information about their education.

6.1.2. Materials and Design.The experiment used a mixed design, with Argument (MP, MT, AC, DA) as a 4-level within subject factor, Rule (causal, means-end) as a 2 level within subject factor), and Defeater (Control, Rebutter, Undercutter) as a 3-level between subject factor. Each participant had to solve four problems, one for each conditional argument, two with a causal rule and another two with a means-end rule, all of them featuring a rebutter, an undercutter, or neither (the control). Order of appearance of the four conditional arguments was counterbalanced in half the questionnaires. Different contents were rotated across problems in order to control for content effects. The four epistemic conditional statements (C1, C2: causal; 
M1, M2: means-end) were the following, translated from French (C1 and C2 express reversed causal relations; M1 and M2 express reversed means end relations):

(C1) If there is snow on the TV screen then there is a storm nearby;

(C2) If Pierre reached Marie's answering machine then Marie was not home;

(M1) If Julie is in the library then she has a presentation to prepare;

(M2) If Sophie takes swimming lessons then she wants to learn swimming.

In line with the overt paradigm, rebutters (RC1, RC2, RM1, and RM2) and undercutters (UC1, UC2, UM1, and UM2) to these relations were framed in conditional form, and were, respectively:

(RC1) If the TV is on then there is snow on the screen;

(RC2) If Marie has an answering machine then Pierre reached Marie's answering machine;

(RM1) If the library is open then Julie is in the library;

(RM2) If Sophie has some spare time, she takes swimming lessons.

(UC1) If the aerial is unplugged then there is snow on the screen;

(UC2) If Marie was filtering her calls then Pierre reached Marie's answering machine;

(UM1) If Julie has some books to borrow then she is in the library; 
(UM2) If Sophie has a crush on the swimming instructor then she takes swimming lessons.

Thus, a MP argument in the Undercutter experimental group, featuring the second means end content, would be formed from premises (M2), (UM2), and 'Sophie takes swimming lessons'; the conclusion to evaluate would be 'she wants to learn swimming'.

Likewise, an AC argument in the Rebutter experimental group, featuring the first causal content, would be formed from premises (C1), (RC1), and 'there is a storm nearby'; the conclusion to evaluate would be 'there is snow on the TV screen'. Each argument was introduced as a conversation between several persons, tupposed to be well informed on the focal character in the argument (i.e., Julie, Marie, or Sophie) - the TV scenario was introduced as a conversation between roommates about the TV of their neighbour, the various technical problems of which they were well informed of.

Participants in the control group had to solve standard arguments. See the Appendix for a detailed example of how a Modus Ponens argument appeared in each experimental group.

In all three groups, participants had to express their confidence in the conclusion of the argument on a 7point scale, according to the following guidelines: ' 1 ' means the conclusion is certainly false, ' 2 ' that it is much more 
likely to be false than to be true, ' 3 ' that it is slightly more likely to be false than to be true, ' 4 ' that it is as likely to be false as to be true, ' 5 ' that it is slightly more likely to be true than to be false, ' 6 ' that it is much more likely to be true than to be false, and ' 7 ' that it is certainly true.

6.1.3. Procedure,Participants were approached in several public places in Paris. Only native French speakers were asked for their participation. The experiment was introduced as a research on reasoning. Participants were told they had to solve four simple problems, which did not include any trick question. They were also told that commonsense was enough to answer the questions, and that they shall not fear to give a seemingly obvious answer, for even obvious answers were of interest to the experimenter.

\subsection{Results and Discussion}

Table 1 displays the mean and standard deviation of the distributions of answers to the four arguments, as a function of Rule and Defeater. All the statistical treatments we will report treat participants' answers as ordinal rankings on a 7 point scale.

We first consider results related to inferential conditionals conveying a causal relation. Results support hypothesis $\mathrm{P} 1$, as confidence in MP conclusions is lower in the Undercutter condition than it is in Control condition, Mann Whitney's $U(N=43)=129.5, p<.01$ (one tailed). Results 
however fail to support hypothesis P2, as confidence in MT conclusions is not reliably lower in the Undercutter condition than it is in Control condition, $U(N=44)=196.5, n s$.

As expected from hypotheses $\mathrm{P} 3$ and $\mathrm{P} 4$, confidence in $\mathrm{AC}$ and DA conclusions is not significantly lower in the Undercutter condition than it is in Control condition, $U(N=43)=182, n s$, for $\mathrm{AC}$, and $U(N=44)=224, n s$, for DA.

Results go nearly against hypothesis P5, as confidence in MP conclusions is almost reliably lower in the Rebutter group than it is in the Control group, $U(N=43)=156.5, p=.06$ (two tailed). Results still support hypothesis P6, as confidence in MT conclusions is not significantly lower in the Rebutter group than it is in the Control group, $U(N=44)=203.5$, ns.

Results do not support hypotheses P7 and P8, as confidence in AC and DA conclusions is not significantly lower in the Rebutter group than it is in the Control group, $U(N=43)=202.5, n s$, for $\mathrm{AC}$, and $U(N=44)=205.5$, $n s$, for DA.

Turning to conditionakfeaturing means-end relations, results again support hypothesis $\mathrm{P}^{\prime}$, as confidence in MP conclusions is lower in the Undercutter condition than it is in Control condition, Mann Whitney's $U$ ( $N$ $=44$ ) $=137.5, p<.01$ (one tailed). Results also support hypothesis $\mathrm{P} 2^{\prime}$, as 
confidence in MT conclusions is reliably lower in the Undercutter condition than it is in Control condition, $U(N=43)=119, p<.01$ (one tailed).

As expected from hypotheses $\mathrm{P}^{\prime}$ and $\mathrm{P}^{\prime}$, confidence in $\mathrm{AC}$ and $\mathrm{DA}$ conclusions is not significantly lower in the Undercutter condition than it is in Control condition, $U(N=44)=192, n s$, for $\mathrm{AC}$, and $U(N=43)=162.5, n s$, for DA.

Results support hypothesis $\mathrm{P5}^{\prime}$ and $\mathrm{P6}^{\prime}$, as confidence in MP and MT conclusions is not reliably lower in the Rebutter group than it is in the Control group, $U(N=44)=174.5, n s$, for MP, and $U(N=43)=167, n s$, for MT.

Results fail to support hypotheses $\mathrm{P}^{\prime}$ and $\mathrm{P} 8^{\prime}$, as confidence in $\mathrm{AC}$ and DA conclusions is not significantly lower in the Rebutter group than it is in the Control group, $U(N=44)=201, n s$, for $\mathrm{AC}$, and $U(N=43)=180, n s$, for DA.

Results of Experiment 1 thus draw a mixed picture. On the one hand, results almost unambiguously support our predictions regarding the effects of undercutters, for causal relations as well as means-end relations: of predictions P1-4 and P1'-4', only prediction P2 fails to get support. On the other hand, our predictions with respect to the effect of rebutters receive weak support, as results only support predictions $\mathrm{P}^{\prime}, \mathrm{P} 6$, and $\mathrm{P} 6^{\prime}$, and give 
tangential support to prediction P5, but fail to support predictions $\mathrm{P} 7, \mathrm{P7}$ ', $\mathrm{P} 8$, and $\mathrm{P} 8$.

Nevertheless, before we rush to conclusions regarding our general hypothesis, we need to control for some pragmatic interference in the data. Experiment 1 introduced undercutters and rebutters in the conditional phrasing that dates back to Rumain et al. (1983) and Byrne (1989). However, as it has been stressed out on a number of occasions (e.g., Bonnefon \& Hilton, 2002; Politzer,2005; Politzer \& Bourmeau, 2002; Politzer \& Braine, 1991; Stevenson \& Over, 1995), this conditional phrasing requires reasoners to compute an implicature to the effect that the undercutter (or the rebutter) it contains in its antecedent may be actual (or active). This pragmatic complication of the task may cause some noise in the data, as we cannot control for the proportion of participants who did not generate the implicature but would have integrated the undercutter or the rebutter into their reasoning, had it been introduced in a more straightforward way.

Therefore, we saw a need to run a pragmatically simplified version of Experiment 1, where undercutters and rebutters would be introduced in a non-conditional manner (e.g., 'It is not certain that the library is open') rather than conditionally (e.g., 'If the library is open then Julie is at the library'). This mode of presentation is also justified by the results of Dieussaert, Schaeken, and d'Ydewalle (2002) who observed, with a variety 
of conditionals, that belief in the conclusion of MP arguments was lower when the additional premise was categorical rather than conditional.

\section{Experiment 2}

\subsection{Method}

7.1.1 Participants. A total of 72 participants were approached in Experiment $2($ mean age $=30.1, S D=14.4)$. Thirty-eight of them had received college education or an equivalent, 20 had graduated from high school without pursuing further education, and 14 had not graduated from high school. 7.1.2 Materials and Design. The design of Experiment 2 was identical to the design of Experiment 1, Argument (MP, MT, AC, DA) being a 4-level withinsubject factor, Rule (causal, means-end) a 2-level within-subject factor), and Defeater (Control, Rebutter, Undercutter) a 3-level between subject factor.

No data collection was actually conducted with respect to the control group, as (a) the control condition was in every respect similar to the control condition in Experiment 1, and (b) participants in Experiment 2 were recruited from the same general population as participants in Experiment 1; thus, the data from the control group in Experiment 1 were re-used in Experiment 2. 
The materials used in Experiment 2 were similar in every respect to the materials used in Experiment 1, except for the phrasing of rebutters and undercutters, which became:

(RC1') It is not certain that the TV set is on,

(RC2') It is not certain that Marie has an answering machine,

(RM1') It is not certain that the library is open,

(RM2') It is not certain that Sophie has time for swimming lessons,

(UC1') The aerial might be unplugged,

(UC2') Marie might have been filtering her calls,

(UM1') Julie might have some books to borrow,

(UM2') Sophie might have a crush on the swimming instructor.

7.1.3 Procedure. Procedure was the same as in Experiment 1.

\subsection{Results and Discussion}

Table 2 displays the mean and standard deviation of the distributions of answers to the four arguments, as a function of Rule and Defeater. All but one of our 16 hypotheses are supported by the results. Considering conditionals featuring causal relations, results support hypothesis P1 and P2, as confidence in MP and MT conclusions is lower in the Undercutter condition than it is in Control condition, Mann Whitney's $U(N=39)=121, p<.05$ (one tailed), for MP, and $U(N=40)=122.5, p<.05$ (one tailed), for MT. 
As expected from hypotheses $\mathrm{P} 3$ and $\mathrm{P} 4$, confidence in $\mathrm{AC}$ and DA conclusions is not significantly lower in the Undercutter condition than it is in Control condition, $U(N=39)=140, n s$, for $\mathrm{AC}$, and $U(N=40)=198, n s$, for DA.

Results also support hypotheses P5 and P6, as confidence in MP and MT conclusions is not reliably lower in the Rebutter group than it is in the Control group, $U(N=39)=134.5, n s$, for $\mathrm{MP}$, and $U(N=40)=174, n s$, for MT.

As opposed to what was observed in Experiment 1, results support hypothesis P7, as confidence in AC conclusions is lower in the Rebutter group than it is in the Control group, $U(N=39)=96.5, p<.01$ (one tailed). Hypothesis P8 is the only one not to receive support from the results, as confidence in DA conclusions is not reliably lower in the Rebutter group than in the Control group, $U(N=40)=160.5, n s$.

All hypotheses related to conditionals featuring a meansend relation receive support from the results. Confidence in the conclusions of MP and MT are reliably lower in the Undercutter group, $U(\mathrm{~N}=40)=124, p<.05$ (one tailed), for MP, and $U(\mathrm{~N}=39)=96.5, p<.01$ (one tailed), for MT. On the contrary, confidence in the conclusions of MP and MT are not reliably lower in the Rebutter group, $U(\mathrm{~N}=40)=185.5, n s$, for $\mathrm{MP}$, and $U(\mathrm{~N}=39)=$ $168, n s$, for MT. 
Symmetrically, confidence in the conclusions of AC and DA are reliably lower in the Rebutter group, $U(\mathrm{~N}=40)=128, p<.05$ (one tailed), for AC, and $U(\mathrm{~N}=39)=128.5, p<.05$ (one tailed), for DA. On the contrary, confidence in the conclusions of AC and DA are not reliably lower in the Undercutter group, $U(\mathrm{~N}=40)=138, n s$, for $\mathrm{AC}$, and $U(\mathrm{~N}=39)=158, n s$, for DA.

Results of Experiment 2 give very strong support to our general hypotheses, as, of 16 experimental predictions, only prediction P8 was not corroborated by the results.

\section{General Discussion}

The results of the experiments show that Cummins's (1995) observation that one can reverse the Core Pattern of defeasible inferences in causal conditional reasoning is robust, as this observation was made initially with the covert paradigm, and we extended it to the overt paradigm. We also tested this reversion beyond the causal domain, namely for meansend rules, and found that it is not limited to causal rules. This is consistent with results already reported in the literature with standard (non-inferential) conditional rules. In some of the studies already mentioned, the role of rebutters and undercutters has been established with non causal rules: Permission and 
precaution (Thompson, 1995); promises, tips, warnings, threats, intentional, temporal, obligation, prediction (Newstead et al., 1997; Evans \& Twyman Musgrove, 1998; Dieussaert, Schaeken, \& d'Ydewalle, 2002).

In brief, a wide variety of relationships gives rise to conclusion retraction in conditional reasoning, and it can be considered as established that this phenomenon is not limited to causality. One can conclude that Cummins's observation seems to be but one case of a more general phenomenon that finds an explanation in Pollock's view of defeaters, a conceptualization that extends beyond the causal domain.

At a descriptive level, this means that people are responsive to logical necessity and sufficiency as well as to causal necessity and sufficiency. Like disablers and alternatives for causal relationships, rebutters and undercutters modulate the degree of necessity and sufficiency of various relationships, including relations of causality. The essential point made by Cummins, which we have confirmed and extended, revolves around the intrinsic definition of defeaters for a given conditional rule, which explains why turning one type of inferential into the other one (e.g., necessaryepistemic into possible-epistemic) preserves the defeaters' effect.

So far, we have not concerned ourselves with the mechanism by which defeaters affect belief in the conclusion. Several approaches to the suppression of sound inferences can be found in the literature mentioned 
earlier. According to the mental model approach, the key factor is the existence of a counterexample to the conclusion of the argument (Byrne, Espino \& Santamaría, 1999); but it has been shown that this approach cannot explain participants' responses in the variant of the overt paradigm that consists of a production task (Politzer, 2005). Even more crucially, it has been argued that it cannot explain participants systematic allocation of degrees of belief to the conclusion as a function of the credibility of the premises in both paradigms (Politzer \& Bourmaud, 2002).

A different approach to conditional reasoning has been developed in the past few years based on Bayesian theory (Liu, Lo \& Wu, 1996; Oaksford, Chater \& Larkin, 2000); this seems to provide an appropriate conceptual tool to study the suppression of inferences, to the extent that this suppression stems from the probabilistic nature of the premises. In line with this approach, the hypothesis that the credibility of natural language conditionals is evaluated by the conditional probability of the consequent given the antecedent has gained much support (Evans, Handley \& Over, 2003; Oberauer \& Wilhelm, 2003; Over \& Evans, 2003). We will briefly show that the present results are compatible with an approach that accommodates Bayesian revision within formal rule theory. We will focus on the MP case which is the most fundamental, and consider rebutters first. 
It is easy to show that, as the probability of a rebutter s being present or active increases, the conditional probability of the major premise decreases. The non occurrence (or the non satisfaction) of a rebutter $\mathrm{R}$ being a necessary condition for the consequent $\mathrm{C}$ of the conditional to occur, it is always the case that:

$$
P(C) \leq P(\neg R)
$$

Moreover, considering that:

$$
P(C \mid A)=\frac{P(C \wedge A)}{P(A)}
$$

And that:

$$
P(A \wedge C) \leq P(C)
$$

It follows that:

$$
P(C \mid A) \leq \frac{P(\neg R)}{P(A)}
$$

Thus, when $\mathrm{P}(\mathrm{A})$ is constant, the conditional probability of $\mathrm{C}$ being true given that $\mathrm{A}$ is true can only decrease as the probability of the rebutter increases. Rebutters act by decreasing the conditional probability of the major premise, and therefore, according to the view under consideration, by decreasing the credibility of this major premise.

One might object that since $P(C)$ is always inferior to $P($ not $R)$, the decrease in the belief of the MP conclusion $C$ is confounded with that of the 
conditional if $A, C$, and the former factor is a more direct explanation of the suppression of MP than the latter factor. This is correct, but it will not do for MT, whose conclusion not A does not depend straightforwardly on R. Considering that if Julie has a presentation to prepare then she is in the library (if $A, C)$, the fact that the library is not open (R) does not have any bearing on her having or not having a presentation to prepare (not A). In that case, belief in $\mathrm{R}$ does not affect the conclusion of the MT argument but rather decreases belief in if $A, C$.

By comparison, how can undercutters defeat the conclusion of a MP argument? More precisely, why is belief in A lower from premises if $C$ then $A$, maybe $U, A$ than from premises if $C$ then $A, A$, where $U$ is another possible reason for $\mathrm{C}$ ? In order to give a Bayesian account of this phenomenon, we need to arrive at the following inequality:

$$
P(C \mid A \wedge U)<P(C \mid A)
$$

It can be shown (see Benferhat, Cayrac, Dubois, \& Prade, 1996, for a complete demonstration) that this inequality is formally equivalent to:

$$
\left.\left.P\left(C \mid A_{\wedge} U\right) \cdot P(C \mid \tau A \wedge\urcorner U\right)<P(C \mid \neg A \wedge U) . P\left(C \mid A_{\wedge}\right\urcorner U\right) .
$$

Now, under the hypothesis that no other reason than $A$ or $U$ is relevant in the context to the realisation of $\mathrm{C}$, that is, that one at least of $\mathrm{A}$ or $\mathrm{U}$ must be true when $C$ is true, this last inequality trivially holds: The left term is 
null since $C$ excludes not $A$ \& not $U$, so that the conditional probability of $C$ given not $A$ and not $U$ is null, and the second term is strictly positive from the definition of both $\mathrm{A}$ and $\mathrm{U}$ as reasons that can make $\mathrm{C}$ happen. This kind of causal structure is known in Artificial Intelligence as 'explaining away' (Pearl, 1988). In such a structure, if two factors A and U are probabilistically independent, they will become negatively correlated when conditioned on an event C. Similarly, in the present case, learning $U$ weakens the probability of A conditioned on $\mathrm{C}$.

In conclusion, we have seen that rebutters act by decreasing the credibility of the major conditional; they do this by weakening the sufficiency of its antecedent for the consequent to occur. Undercutters act in a different manner: Although they also act by decreasing the credibility of the major conditional, they do so by weakening the necessity of its consequent. In agreement with Pollock (1987) they can act so radically as to give a reason to deny the conditional altogether by replacing it with an alternative conditional. However, our conclusion is slightly at variance with his characterization of how rebutters act: He argued that they affect directly the conclusion of the argument, whereas our analysis, which includes the case of MT arguments, leads us to conclude that rebutters affect the conditional (as undercutters do, but by a different mechanism). 
We remarked earlier that necessary-epistemic inferentials are linked to demonstrative inference, whereas possible-epistemic inferentials are linked to non demonstrative inference. While the former constitute some kind of reduced Modus Ponens, we now propose a parallel characterization for the latter. Consider the sentences from (10) to (12) and from $\left(10^{\prime}\right)$ to $\left(12^{\prime}\right)$ above. Whereas a necessary epistemic inferential can be regarded as the embedding of the minor premise and the conclusion of an MP argument within an 'if...then' frame, a possible epistemic inferential can be regarded as the embedding of the minor premise and the conclusion of an $\mathrm{AC}$ argument, of which the virtual major premise would be the associated necessary-epistemic conditional. Formally, this is what gives the consequent of the possible-epistemic inferential its non demonstrative character, as the $\mathrm{AC}$ inference has no deductive validity.

More precisely, we propose that a more serious, positive (and also charitable) view be taken on the use of $\mathrm{AC}$ (and DA) arguments; that is, psychologists should break with an old logical tradition by which these arguments are considered exclusively from the point of view of demonstrative inference, which turns their use into deductive fallacies. Rather, their use should be acknowledged as an invaluable source of non demonstrative inference in human reasoning. (It is also suggested, for methodological reasons, that their study should be kept carefully away from 
human deduction.) There are, in fact, a number of logicians and philosophers of the past who paid heed to AC (starting with Aristotle himself, who considered, in the Prior Analytics, the syllogistic equivalent of the $\mathrm{AC}$ argument, sometimes called reductio and sometimes abductio in the latin version of his writings). Pierce's (1935 1958) conception of the role of $\mathrm{AC}$ (which he designated by the term of abduction in his early writings) has been theoretically decisive, but has had little impact among psychologists. Yet, inferring from a fact $\mathrm{F}$ to a reason for $\mathrm{F}$, which is pervasive in daily life, can be described by the search for a conditional relation 'if $R$ then $F^{\prime}$, yielding $R$ as a plausible reason that explains $F$.

It is therefore no wonder that the use of the two fundamental arguments by which people draw inferences to particular (or singular) propositions, namely $\mathrm{MP}$ and $\mathrm{AC}$, can be linguistically signalled to an interlocutor by a special device, which is what the two varieties of inferential conditionals consist in. That is, an inferential conditional can be put to two uses, both of which exploit the same general fundamental property of conditional sentences, namely licence MP: One (in the necessary-epistemic case) to make a deductive inference by a 'compacted' MP (if cause then effect, where cause is affirmed and effect is inferred); the other, more subtly (in the possible-epistemic case), to make an abductive inference by a hidden 'compacted' AC argument in the guise of an MP argument (if effect then 
cause, where effect is affirmed and cause is inferred), which is made possible by the fundamental property just reminded. Thus, human abductive reasoning can be performed by the exploitation of a formally entirely deductive derivation, but one in which a premise is uncertain, that is, a plausible reasoning process (defined as the use of a deductively valid argument in which at least one premise is uncertain. We thus concur with Walton's (2004) 'forward scheme of abductive inference' that uses a defeasible MP. 


\section{References}

Benferhat, S., Cayrac, D., Dubois, D. and Prade, H. 1996: Explaining away in a possibilistic setting. In Proceedings of the 6th International Conference on Information Processing and Management of Uncertainty in Knowledge Based Systems (IPMU 96), Granada, Spain.

Bonnefon, J. F. and Hilton, D. J. 2002: The suppression of Modus Ponens as a case of pragmatic preconditional reasoning. Thinking $\mathcal{E}$ Reasoning, 8, 21 40.

Bonnefon, J. F. and Hilton, D. J. 2004: Consequential conditionals: Invited and suppressed inferences from valued outcomes. Journal of Experimental Psychology: Learning, Memory, and Cognition, 30, 2837.

Byrne, R. M. J. 1989: Suppressing valid inferences with conditionals. Cognition, 31, 6183.

Byrne, R. M. J., Espino, O. and Santamaría, C. 1999: Counterexamples and the suppression of inferences. Journal of Memory and Language, 40, 34773.

Chan, D. and Chua, F. 1994: Suppression of valid inferences: Syntactic views, mental models, and relative salience. Cognition, 53, 21738.

Cummins, D. D. 1995: Naïve theories and causal deduction. Memory $\mathcal{E}$ Cognition, 23, 64658 . 
Cummins, D. D., Lubart, T., Alksnis, O. and Rist, R. 1991: Conditional reasoning and causation. Memory $\mathcal{E}$ Cognition, 19, 27482.

Dancygier, B. 1993: Interpreting conditionals: Time, knowledge, and causation. Journal of Pragmatics, 19, 40334.

Dancygier, B. 1998: Conditionals and Prediction. Time, Knowledge, and Causation in Conditional Constructions. Cambridge: Cambridge University Press.

De Neys, W., Schaeken, W. and d'Ydewalle, G. 2002: Causal conditional reasoning and semantic memory retrieval: A test of the semantic memory framework. Memory E Cognition, 30, 90820.

De Neys, W., W. Schaeken and G. d'Ydewalle 2003: Inference suppression and semantic memory retrieval: Every counterexample counts. Memory $\mathcal{E}$ Cognition, 31, 58195.

Dieussaert, K., Schaeken, W. and d'Ydewalle G. 2002: The relative contribution of content and context factors on the interpretation of conditionals. Experimental Psychology, 49, 18195.

Dieussaert, K., Schaeken, W., Schroyens, W. and d'Ydewalle, G. 2000: Strategies during complex deductive inferences. Thinking and Reasoning, 6,12560 .

Evans, J. St. B. T. and Twyman Musgrove, J. 1998: Conditional reasoning with inducements and advice. Cognition, 69, B11 B16. 
Evans, J. St. B. T. , Handley, S. J. and Over, D. E. 2003: Conditionals and conditional probability. Journal of Experimental Psychology: Learning, Memory, and Cognition, 29, 32135.

Liu, I. M. , Lo, K. C and Wu, J. T. 1996: A probabilistic interpretation of If Then . Quarterly Journal of Experimental Psychology, 49A, 82844.

Manktelow, K. and Fairley, N. 2000: Superordinate principles in reasoning with causal and deontic conditionals. Thinking and Reasoning, 6, 4165.

Markovits, H. 1986: Familiarity effects in conditional reasoning. Journal of Educational Psychology, 78, 49294.

Markovits, H. and Potvin, F. 2001: Suppression of valid inferences and knowledge structures: The curious effect of producing alternative antecedents on reasoning with causal conditionals. Memory $\mathcal{E}$ Cognition, 29,73644 .

Markovits, H. and Quinn, S. 2002: Efficiency of retrieval correlates with 'logical' reasoning from causal conditional premises. Memory $\mathcal{E}$ Cognition, 30, 696706.

Newstead, S. E., Ellis, M. C., Evans, J. St. B. T. and Dennis, I. 1997: Conditional reasoning with realistic material. Thinking and Reasoning, 3, 4976.

Oaksford, M. and Chater, N. 2003: Probabilities and pragmatics in conditional inference: Suppression and order effects. In D. Hardman \& 
L. Macchi (eds.) Thinking: Psychological Perspectives on Reasoning, Judgment, and Decision Making. Chichester, England: Wiley.

Oaksford, M. , Chater, N. and Larkin, J. 2000: Probabilities and polarity biases in conditional inference. Journal of Experimental Psychology: Learning, Memory, and Cognition, 26, 88399.

Oberauer, K. and Wilhelm, O. 2003: The meaning(s) of conditionals: Conditional probabilities, mental models, and personal utilities. Journal of Experimental Psychology: Learning, Memory, and Cognition, 29, 68093.

Over, D. E. and Evans, J. St B. T. 2003: The probability of conditionals: the psychological evidence. Mind and Language, 18, 34058.

Pearl, J. 1988: Probabilistic Reasoning in Intelligent Systems. San Mateo, CA: Morgan Kaufmann.

Peirce, C. S. 1935 1958: Collected Papers. C. Harstone and P. Weiss, eds. Cambridge: Harvard University Press.

Politzer, G. 2005: Uncertainty and the suppression of inferences. Thinking and Reasoning, 11, 533.

Politzer, G. and Bourmeau G. 2002: Deductive reasoning from uncertain conditionals. British Journal of Psychology, 93, 34581.

Politzer, G. and Braine, M. D. S. 1991: Responses to inconsistent premises cannot count as suppression of valid inferences. Cognition, 38, 10308. Pollock, J. L. 1987: Defeasible Reasoning. Cognitive Science, 11, 481518. 
Quinn, S. and Markovits, H. 1998: Conditional reasoning, causality, and the structure of semantic memory: Strength of association as a predictive factor for content effects. Cognition, 68, B93 B101.

Rumain, B., Connell, J. and Braine, M. D. S. 1983: Conversational processes are responsible for reasoning fallacies in children as well as adults: If is not the biconditional. Developmental Psychology, 19, 47181.

Stevenson, R. J. and Over, D. E. 1995: Deduction from uncertain premises. Quarterly Journal of Experimental Psychology, 48A, 61343.

Sweetser, E. E. 1990: From Etymology to Pragmatics. Cambridge: Cambridge University Press.

Thompson, V. A. 1994: Interpretational factors in conditional reasoning. Memory \& Cognition, 22, 74258.

Thompson, V. A. 1995: Conditional reasoning: The necessary and sufficient conditions. Canadian Journal of Experimental Psychology, 49, 160.

Walton, D. 2004: Abductive reasoning. Tuscaloosa: University of Alabama Press. Werth, P. 1997: Conditionality as cognitive distance. In A. Athanasiadou and R. Dirven (eds) On conditionals again. Amsterdam: John Benjamins. 


\section{Appendix}

As an example of the problems involved in the experimental task, we detail in this appendix how the premises of a given Modus Ponens argument appeared in each of the experimental groupsin Experiment 1. In the three groups, the conclusion to be evaluated was 'Sophie wants to learn swimming'.

Control group

If Sophie takes swimming lessons, then she wants to learn swimming; Sophie takes swimming lessons.

\section{Undercutter group}

If Sophie takes swimming lessons, then she wants to learn swimming;

If Sophie has a crush on the swimming instructor, then she takes swimming lessons;

Sophie takes swimming lessons.

Rebutter group

If Sophie takes swimming lessons, then she wants to learn swimming;

If Sophie has some spare time, she takes swimming lessons;

Sophie takes swimming lessons. 
Table 1

Experiment 1: Mean and Standard Deviation of the Distributions of Answers (on a 7 point Scale) to the Four Conditional Arguments, as a function of Type of Rule and Type of Conditional Defeater.

\begin{tabular}{lcccc}
\hline & MP & MT & AC & DA \\
\hline Causal & & & & \\
Control & $5.6(2.0)$ & $4.6(1.6)$ & $5.8(1.9)$ & $5.0(1.5)$ \\
Rebutter & $4.4(1.6)$ & $4.9(1.8)$ & $5.5(1.7)$ & $4.4(1.8)$ \\
Undercutter & $4.2(2.1)$ & $4.0(1.6)$ & $5.1(1.8)$ & $5.0(1.8)$ \\
Means end & & & & \\
Control & $6.0(1.2)$ & $5.5(2.1)$ & $5.5(1.7)$ & $4.9(1.9)$ \\
Rebutter & $5.4(1.3)$ & $4.6(1.3)$ & $5.2(1.4)$ & $4.2(1.8)$ \\
Undercutter & $4.8(1.6)$ & $4.1(2.1)$ & $5.0(1.6)$ & $3.9(1.6)$ \\
\hline
\end{tabular}


Table 2

Experiment 2: Mean and Standard Deviation of the Distributions of Answers (on a 7 point Scale) to the Four Conditional Arguments, as a function of Type of Rule and Type of Unconditional Defeater.

\begin{tabular}{lcccc}
\hline & MP & MT & AC & DA \\
\hline Causal & & & & \\
Control & $5.6(2.0)$ & $4.6(1.6)$ & $5.8(1.9)$ & $5.0(1.5)$ \\
Rebutter & $4.6(1.6)$ & $4.4(1.5)$ & $4.1(1.8)$ & $4.4(1.2)$ \\
Undercutter & $4.6(1.8)$ & $3.3(1.9)$ & $5.4(1.4)$ & $5.0(1.5)$ \\
Means end & & & & \\
Control & $6.0(1.2)$ & $5.5(2.1)$ & $5.5(1.7)$ & $4.9(1.9)$ \\
Rebutter & $5.3(1.4)$ & $4.9(1.5)$ & $4.6(1.3)$ & $3.7(2.1)$ \\
Undercutter & $5.0(1.5)$ & $4.1(2.2)$ & $5.4(1.4)$ & $4.7(1.1)$ \\
\hline
\end{tabular}

